\title{
Intermediate Results of iStent or iStent inject Implantation Combined with Cataract Surgery in a Real-World Setting: A Longitudinal Retrospective Study
}

\author{
Ricardo Augusto Paletta Guedes - Daniela Marcelo Gravina • \\ Jonathan Clive Lake $\cdot$ Vanessa Maria Paletta Guedes $\cdot$ Alfredo Chaoubah
}

Received: December 12, 2018 / Published online: February 5, 2019

(C) The Author(s) 2019

\section{ABSTRACT}

Introduction: In this real-world, retrospective, comparative study we evaluated 6-month performance and safety in consecutive eyes following implantation of the iStent ${ }^{\circledR}$ or iStent inject ${ }^{\circledR}$ trabecular micro-bypass device with concomitant cataract surgery.

Methods: Performance outcomes included intraocular pressure (IOP) reduction; glaucoma medication reduction; proportions of eyes achieving an IOP of $<18,<16,<14$, or $<12 \mathrm{mmHg}$; and proportions of eyes on 0,1 , 2 , or $\geq 3$ medications. Safety outcomes included adverse events, secondary surgeries, and best-corrected visual acuity (BCVA).

Results: A total of 73 eyes with open-angle glaucoma and cataract were included in the study; of these, 38 eyes were implanted with the

Enhanced digital features To view enhanced digital features for this article go to https://doi.org/10.6084/ m9.figshare.7618775.

\footnotetext{
R. A. P. Guedes $(\bowtie) \cdot$ D. M. Gravina .

V. M. P. Guedes

Paletta Guedes Eye Institute, Juiz de Fora, MG, Brazil

e-mail: palettaguedes@yahoo.com

R. A. P. Guedes · A. Chaoubah

Federal University of Juiz de Fora, Juiz de Fora, MG, Brazil

J. C. Lake

Cataract and Glaucoma Department, Brasilia Vision

Hospital, Brasilia, DF, Brazil
}

iStent device and 35 were implanted with the iStent inject device. The two groups of patients had similar baseline characteristics, with the exception of mean age and medication burden (both higher in patients receiving the iStent inject device); over $90 \%$ of eyes in both groups had early glaucoma. At 6 months after surgery, mean IOP had fallen from $16.5 \pm 3.9$ to $13.9 \pm 2.3 \mathrm{mmHg}$ in eyes with the iStent implant $(p<0.001)$, and from $17.3 \pm 3.0$ to $12.7 \pm 1.8 \mathrm{mmHg}$ in those with the iStent inject implant $(p<0.001)$. This reduction was significantly greater in the iStent inject eyes than in the iStent eyes ( 26.6 vs. $15.8 \%)(p=0.005)$. Significantly more eyes receiving the iStent inject device compared to the iStent device achieved an IOP of $<18 \mathrm{mmHg}$ at 6 months post surgery (100 vs. $86.8 \%)(p=0.033)$. Average medication usage was reduced from 1.8 to 0.4 medications in iStent eyes $(p<0.001)$ and from 2.3 to 0.4 medications in iStent inject eyes $(p<0.001)$. Over $70 \%$ of eyes in both groups became medication-free by 6 months post implantation. Adverse events in iStent eyes were mild and resulted in no sequelae; two iStent eyes underwent non-penetrating deep sclerectomy during follow-up. No complications or secondary surgeries were noted in iStent inject eyes. All eyes in both groups maintained or showed improved BCVA versus baseline.

Conclusion: Significant and safe IOP and medication reductions were observed after iStent or iStent inject implantation with concomitant 
cataract surgery. Trends toward greater effectiveness and fewer adverse events were observed with the iStent inject device compared with the iStent device.

Funding: Article processing charges were provided by Glaukos Corporation.

Keywords: Cataract; Glaucoma; Intraocular pressure; iStent; iStent inject; Microinvasive glaucoma surgery; Second-generation; Stent; Trabecular micro-bypass

\section{INTRODUCTION}

The permanent optic nerve damage associated with glaucoma makes it the leading cause of irreversible blindness worldwide. All existing therapies, both medical and surgical, aim to lower intraocular pressure (IOP), which remains the primary risk factor linked to glaucoma progression and visual field decline. Indeed, considerable data support the strong relationship between reduced IOP and reduced glaucoma progression and vision loss [1-4]; this was quantified in the landmark Early Manifest Glaucoma Trial (EMGT) to be approximately $10 \%$ decreased risk of glaucoma progression per every $1 \mathrm{mmHg}$ IOP reduction [1].

Historically, the glaucoma treatment landscape has consisted of medications and/or laser trabeculoplasty as initial treatment, while incisional filtering surgeries, such as trabeculectomy, non-penetrating deep sclerectomy, and tube implants, have rounded out the more invasive end of the treatment spectrum [5]. The effectiveness of medications may be limited by local and systemic side effects, poor adherence rates, difficulty with instillation, complex dosing regimens, and ocular surface hypersensitivities [6-10], while the utility of laser trabeculoplasty is curbed by its waning treatment effect over time [11]. Filtering surgeries yield considerable IOP reduction, but carry risks that include hypotony, infection, bleb-related complications, and/or choroidal detachment [12-14]. Over the past two decades, micro-invasive glaucoma surgery (MIGS) has gained an increasing role in glaucoma treatment, and it may be particularly useful in patients whose disease lies between the aforementioned two extremes on the treatment spectrum.

A substantial body of peer-reviewed evidence has been amassed on the iStent implant (Glaukos Corp., San Clemente, CA, USA), which is the first MIGS device to be approved by the U.S. Food and Drug Administration, and its more recent iteration, the iStent inject implant (Glaukos Corp.) [15-43]. These studies have demonstrated sizable, durable reductions in IOP and medication burden, combined with favorable safety. Results have been achieved in a variety of clinical settings, including with and without cataract surgery, in mild to severe glaucoma, in primary open-angle glaucoma as well as pseudoexfoliative glaucoma and ocular hypertension, in controlled clinical trials and single-surgeon case series, in comparative and non-comparative studies, and in evaluations of single or multiple stents.

Either the iStent (containing one stent) or iStent inject (containing two stents, each with the newer design) devices (Fig. 1) were implanted in the eyes included in the present study. Both devices are designed to decrease IOP by creating a patent pathway for aqueous humor to exit the anterior chamber through the
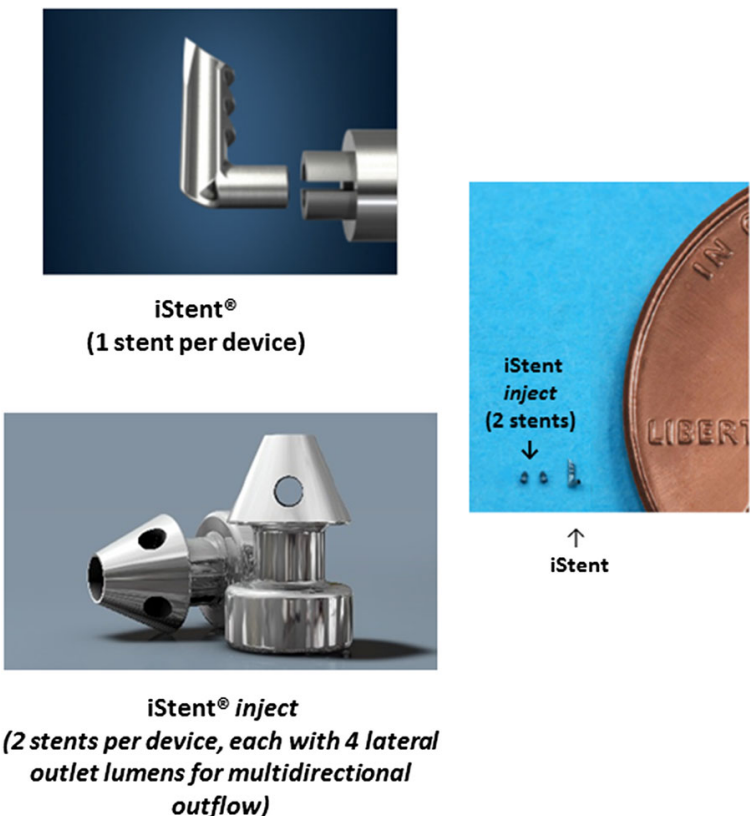

$\uparrow$ iStent

Fig. 1 The iStent ${ }^{\circledR}$ and iStent ${ }^{\circledR}$ inject trabecular microbypass stents, with relative dimensions 
trabecular meshwork into Schlemm's canal. The inclusion of both devices in the cohort allowed us to compare not only one versus two stents, but more importantly to assess the additional benefit of the iStent inject device itself. Such additional benefit may emerge due to the presence of four lateral outlet lumens per stent to allow aqueous fluid to exit from the anterior chamber, potentially maximizing the number of clock-hours of outflow, and also due to the device's greater procedural efficiency, which eases the learning curve for surgeons and conceivably results in more straightforward, complication-free implantation.

The rationale behind the use of multiple versus single stents evolved from preclinical and laboratory studies to clinical trials and singlesurgeon case series. Laboratory investigations by Hunter et al. [44] and Bahler et al. [45, 46], for example, and supportive biomechanical models by Johnstone et al. [47], have demonstrated decreased outflow resistance and reduced IOP after single and double stent placement. In these studies, the bulk of IOP and resistance reduction was achieved after the first stent, with incremental benefit achieved with additional stent placement.

Clinical studies have mirrored these preclinical findings. A prospective randomized trial by Katz et al. [33] compared one, two, and three iStents in a standalone procedure (i.e., without concomitant cataract surgery), and Belovay et al. [39] and El Wardani et al. [40] evaluated two or three iStents during cataract surgery. The results of these three studies confirmed that most IOP reduction is owed to the first stent and that each additional stent yields further IOP and medication reduction.

The present study represents a natural evolution from these prior investigations, with side-by-side outcomes of both the iStent and iStent inject devices in a clinical setting. In this study we assessed the safety and effectiveness outcomes during 6 months following the implantation of either the iStent or iStent inject device in combination with cataract surgery in eyes with mild to moderate open-angle glaucoma. To our knowledge, this is the first study with head-to-head data on the two devices, as observed in a real-world setting in the hands of a single surgeon at a single site.

\section{METHODS}

In this longitudinal retrospective study, we evaluated all consecutive eyes implanted with either the iStent or iStent inject device with concomitant cataract surgery from June 2017 to April 2018. All surgeries were performed in a single center by the same surgeon (R.G.). The choice between implants was based on their availability in the Brazilian market. From June 2017 to November 2017, only the iStent trabecular bypass was commercially available for use, while use of the iStent inject device steadily increased after its approval by the Brazilian regulatory agency in November 2017. Since the level of experience can confound surgical outcomes, we excluded the first ten iStent cases and the first five iStent inject cases from this eye cohort. Thus, all eyes included in this study were unaffected by the surgeon's learning curve.

Eligible patients were over 18 years of age and had open-angle glaucoma, cataract requiring surgery, and the need for IOP and/or medication reduction; follow-up data were available for the entire 6 months following surgery. Eyes with missing data, shorter follow-up than 6 months, and/or significant coexisting ocular diagnoses that could confound the effect of the surgery were excluded.

Main effectiveness outcomes included percentage IOP reduction, percentage medication reduction, and proportion of patients achieving different levels of IOP control at the end of the follow-up (IOP $<18 \mathrm{mmHg}$, $<16 \mathrm{mmHg}$, $<14$ $\mathrm{mmHg}$, or $<12 \mathrm{mmHg}$ ). Safety outcomes included adverse events, secondary surgical interventions, and best-corrected visual acuity (BCVA). IOP was measured by Goldmann applanation, and BCVA was measured using a standard Snellen chart. Proper positioning of the iStent and iStent inject was verified by gonioscopy examination, which was performed both during surgery and at all clinical examinations throughout the follow-up.

Chi-square test and Student's $t$ test were used for the analysis of categorical and numerical 
variables, respectively. Statistical analysis was performed using SPSS statistical software (IBM Corp., Armonk, NY, USA), with a $p$ value of 0.05 as the threshold for statistical significance.

All procedures were in accordance with the Institutional Review Board of the Paletta Guedes Eye Institute and with the 1964 Helsinki declaration and its later amendments or comparable ethical standards. Given that this was a retrospective outcomes analysis that included only patients from the surgeon's real-world clinical population who already had received treatment, formal clinical trial registration was not required. As it was a retrospective study based on information on the patients' medical records, informed consent from study participants was not necessary.

\section{RESULTS}

\section{Demographics and Baseline Ocular Characteristics}

A total of 73 eyes met inclusion criteria and comprised the study population. These included 38 eyes receiving an iStent implant and 35 eyes receiving an iStent inject for which 6 months of postoperative follow-up data were available. The mean \pm standard deviation age was $70.9 \pm 9.0$ years. The majority of patients were female $(65.8 \%)$. Glaucoma stage was early, moderate and advanced in 97.3, 1.4, and 1.4\% of study eyes, respectively.

Table 1 presents the preoperative comparison between groups. Groups were statistically comparable in baseline IOP, laterality (right or left eye), glaucoma stage, and baseline visual acuity. The two implant groups differed in their mean age (patients receiving the iStent inject device were an average of 8 years older) and the number of medications at baseline (patients receiving the iStent inject device had a higher mean number of medications).

\section{Intraocular Pressure}

Mean IOP in the iStent group was reduced from $16.5 \pm 3.9 \mathrm{mmHg}$ at baseline to
Table 1 Demographic and baseline ocular characteristics according to the iStent and iStent inject groups

\begin{tabular}{|c|c|c|c|}
\hline $\begin{array}{l}\text { Patient } \\
\text { characteristics }\end{array}$ & $\begin{array}{l}\text { iStent } \\
\text { device } \\
(n=38 \\
\text { eyes })\end{array}$ & $\begin{array}{l}\text { iStent } \\
\text { inject } \\
\text { device } \\
(n=35 \\
\text { eyes })\end{array}$ & $\begin{array}{l}p \text { value } \\
\text { (between- } \\
\text { group } \\
\text { comparison) }\end{array}$ \\
\hline Age (years) & $67.1 \pm 8.8$ & $75.1 \pm 7.1$ & $<0.001^{\mathrm{a}}$ \\
\hline $\begin{array}{c}\text { Baseline IOP } \\
(\mathrm{mmHg})\end{array}$ & $16.5 \pm 3.9$ & $17.3 \pm 3.0$ & $0.275^{\mathrm{a}}$ \\
\hline $\begin{array}{l}\text { Baseline } \\
\text { number of } \\
\text { medications }\end{array}$ & $1.8 \pm 0.9$ & $2.3 \pm 1.0$ & $0.019^{\mathrm{a}}$ \\
\hline \multicolumn{4}{|l|}{ Race } \\
\hline Caucasian & $60.5 \%$ & $82.6 \%$ & $0.122^{\mathrm{b}}$ \\
\hline $\begin{array}{l}\text { African } \\
\text { descent }\end{array}$ & $39.5 \%$ & $17.4 \%$ & \\
\hline \multicolumn{4}{|l|}{ Gender } \\
\hline Male & $28.9 \%$ & $40.0 \%$ & $0.227^{\mathrm{b}}$ \\
\hline Female & $71.1 \%$ & $60.0 \%$ & \\
\hline \multicolumn{4}{|l|}{ Glaucoma stage } \\
\hline Early & $100 \%$ & $94.2 \%$ & $0.189^{\mathrm{b}}$ \\
\hline Moderate & $0.0 \%$ & $2.9 \%$ & \\
\hline Advanced & $0.0 \%$ & $2.9 \%$ & \\
\hline \multicolumn{4}{|c|}{ Baseline visual acuity } \\
\hline $\begin{array}{l}20 / 30 \text { or } \\
\text { better }\end{array}$ & $47.4 \%$ & $34.3 \%$ & $0.101^{b}$ \\
\hline $\begin{array}{l}20 / 40 \text { up to } \\
\text { (but not } \\
\text { including) } \\
20 / 200\end{array}$ & $52.6 \%$ & $57.1 \%$ & \\
\hline $\begin{array}{l}20 / 200 \text { or } \\
\text { worse }\end{array}$ & $0.0 \%$ & $8.6 \%$ & \\
\hline
\end{tabular}

Values in table are presented as the mean \pm standard deviation $(\mathrm{SD})$ or as the percentage, as appropriate $I O P$ Intraocular pressure

a Student's $t$ test

b Chi-square test 
$13.9 \pm 2.3 \mathrm{mmHg}$ at 6 months post implantation $(p<0.001)$. In the iStent inject group, mean IOP dropped from $17.3 \pm 3.0$ at baseline to $12.7 \pm 1.8 \mathrm{mmHg}$ at 6 months post implantation $(p<0.001)$. Mean percentage IOP reduction was significantly greater in eyes receiving the iStent inject device (26.6\%) than in those receiving the iStent implant (15.8\%) $(p=0.005)$.

Figure 2 shows the mean IOP through the 6 months of follow-up for the overall eye cohort. Figure 3 and Table 2 show the mean IOP through the 6 months of follow-up for each study group separately. Statistically significant reductions in IOP versus baseline were achieved at all time points in the iStent inject group, and at all but two time points (Days 15 and 30) in the iStent group. IOP distribution into different IOP thresholds at the end of follow-up is shown in Table 3. Although baseline IOP was statistically similar between groups, at
6 months post surgery, significantly more iStent inject eyes than iStent eyes achieved an IOP of $<18 \mathrm{mmHg} \quad(p=0.033)$ and IOP of $<12 \mathrm{mmHg}(p=0.04)$. More eyes with the iStent inject implant also achieved the other IOP thresholds at 6 months, but the between-group difference was not statistically significant at these latter thresholds ( 84.2 and $55.3 \%$ of iStent eyes vs. 88.6 and $71.4 \%$ of iStent inject eyes achieved a final IOP $<16$ and $<14 \mathrm{mmHg}$, respectively; $p>0.05)$.

\section{Medications}

In eyes implanted with the iStent device, the mean number of medications reduced from 1.8 at baseline to 0.4 medications at 6 months post surgery $(77.8 \%$ reduction; $p<0.001)$; in eyes implanted with the iStent inject device, medication usage reduced from 2.3 to 0.4 medications over the same time period $(82.6 \%$

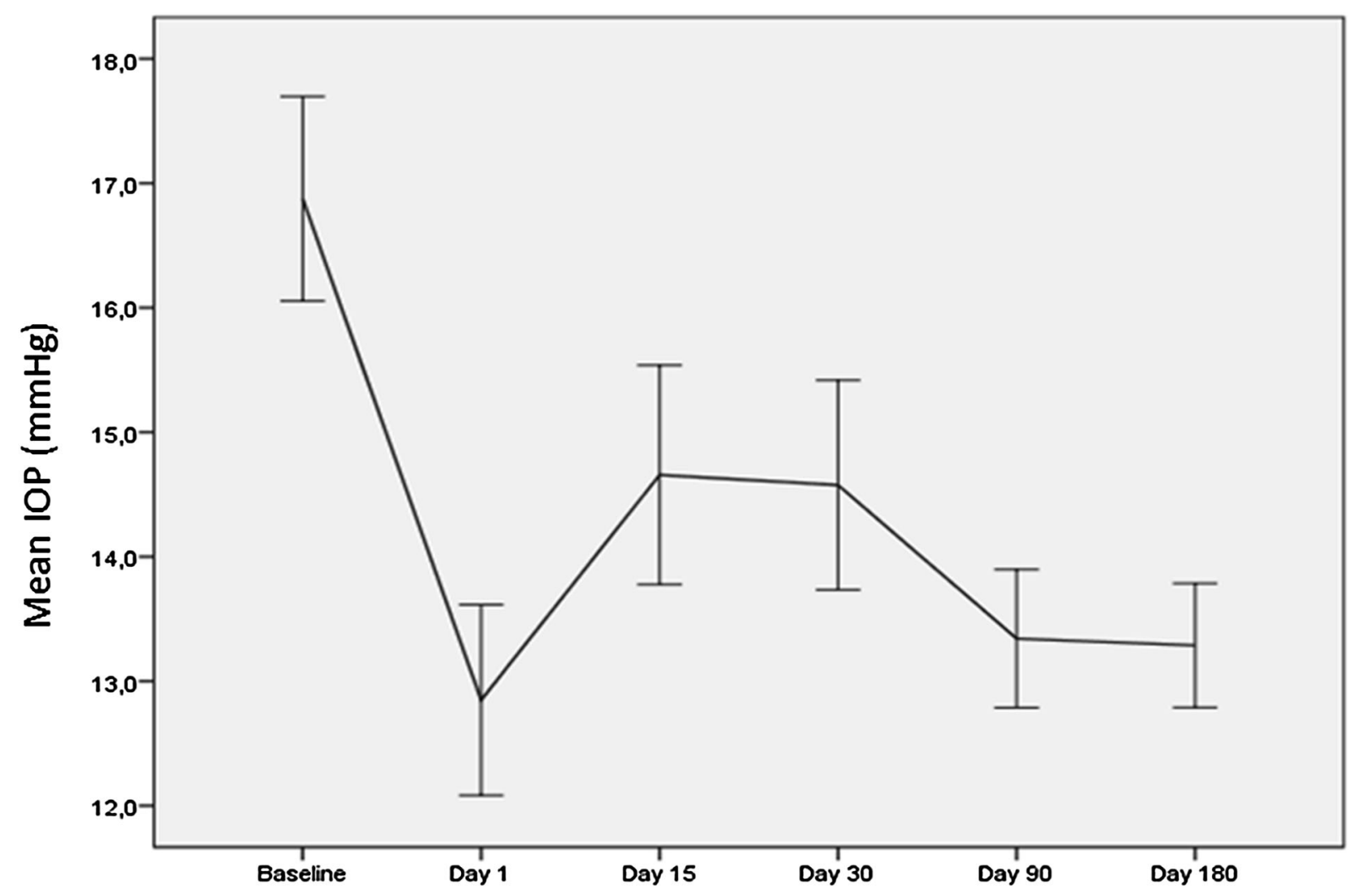

Fig. 2 Mean intraocular pressure (IOP) from baseline to 6 months (Day 180) post surgery in the overall cohort $(n=73$ eyes). $p<0.001$ at all postoperative visits. Vertical bars represent the standard error (SE) 


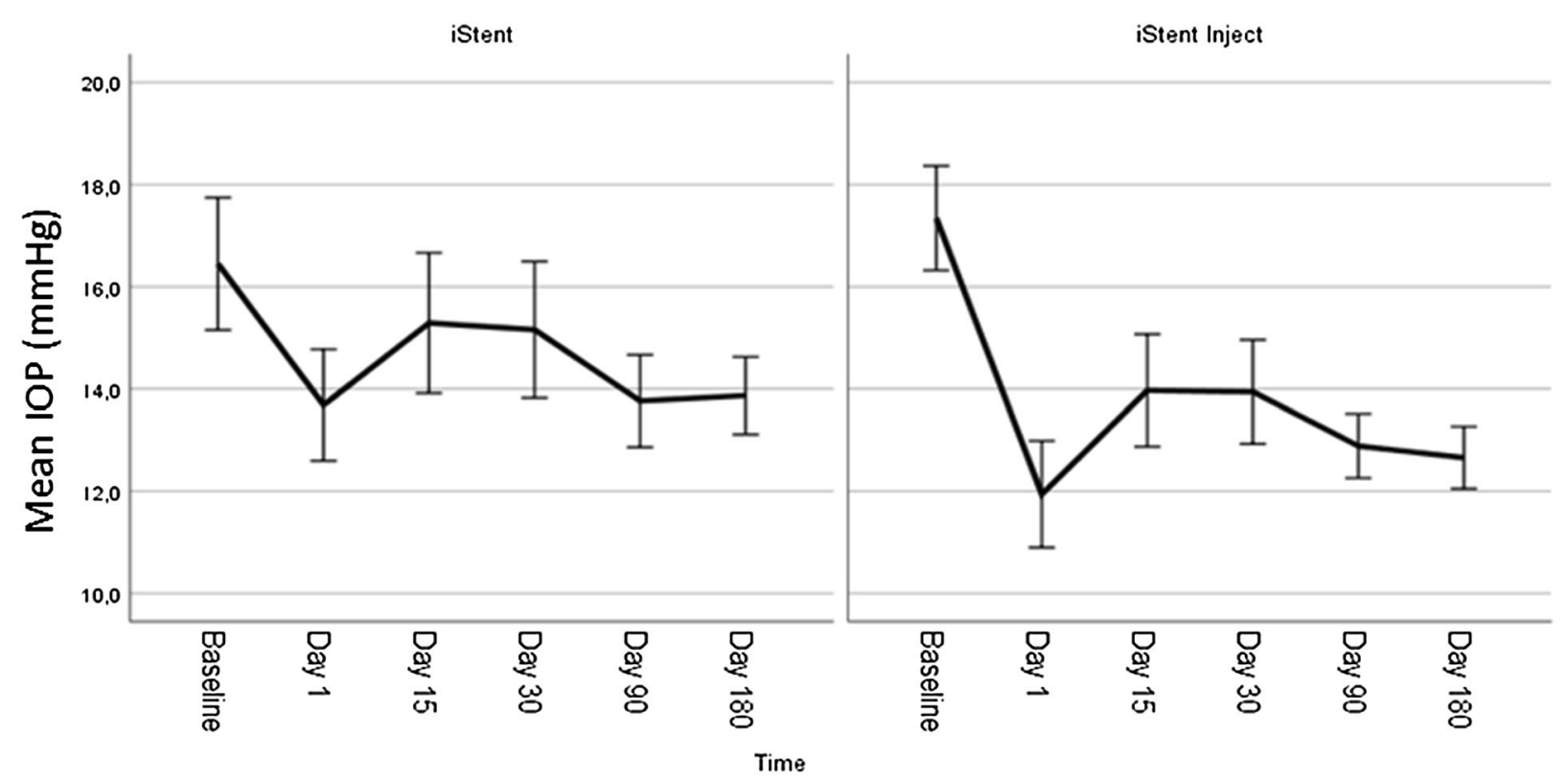

Fig. 3 Mean IOP from baseline to 6 months (Day 180) post surgery according to type of implant (iStent, $n=38$ eyes; iStent inject, $n=35$ eyes). $p<0.001$ at all postoperative visits in eyes with the iStent inject device, and at all but two visits (Days 15 and 30) in eyes with the iStent device. Vertical bars represent SE

Table 2 Intraocular pressure from baseline to 6 months post surgery according to type of implant

\begin{tabular}{llll}
\hline $\begin{array}{l}\text { Time } \\
\text { Point }\end{array}$ & $\begin{array}{l}\text { IOP }(\mathbf{m m H g}), \text { iStent device } \\
(\boldsymbol{n}=\mathbf{3 8} \text { eyes})^{\mathbf{a}}\end{array}$ & $\begin{array}{l}\text { IOP }(\mathbf{m m H g}) \text { iStent } \text { inject device } \\
(\boldsymbol{n}=\mathbf{3 5} \text { eyes })^{\mathbf{a}}\end{array}$ & $\begin{array}{l}\boldsymbol{p} \text { value } \mathbf{b} \text { (between-group } \\
\text { comparison of IOP at each } \\
\text { time point })\end{array}$ \\
\hline Baseline & $16.5 \pm 3.9$ & $17.3 \pm 3.0$ & 0.275 \\
Day 1 & $13.7 \pm 3.3(p=0.001)$ & $11.9 \pm 3.0(p<0.001)$ & 0.022 \\
Day 15 & $15.3 \pm 4.2(p=0.135)$ & $14.0 \pm 3.2(p<0.001)$ & 0.133 \\
Day 30 & $15.2 \pm 4.1(p=0.074)$ & $13.9 \pm 3.0(p<0.001)$ & 0.152 \\
Month 3 & $13.8 \pm 2.8(p<0.001)$ & $12.9 \pm 1.8(p<0.001)$ & 0.116 \\
Month 6 & $13.9 \pm 2.3(p<0.001)$ & $12.7 \pm 1.8(p<0.001)$ & 0.014 \\
\hline
\end{tabular}

Values in table are presented as the mean $\pm S D$

a Paired Student's $t$ test (comparison vs. baseline); i.e., $p$ value in each cell for IOP at that time point vs. baseline

b Student's $t$ test (comparison between groups)

reduction; $p<0.001$ ) (Fig. 4). Prior to surgery (i.e. at baseline) 63.1 and $74.3 \%$ of eyes in the iStent and iStent inject groups, respectively, were on $\geq 2$ glaucoma medications, and 5.3 and $0 \%$ of eyes, respectively, were medicationfree (Fig. 5a, b). By 6 months post surgery, a similarly high number of eyes in both groups had become medication-free $(71.1 \%$ in iStent eyes and $74.3 \%$ in iStent inject eyes; $p=0.482$ ) (Fig. $5 \mathrm{a}, \mathrm{b}$ ). The medication regimen postoperative was either decreased or maintained versus the preoperative (baseline) regimen for all eyes in both implant groups. 
Table 3 Proportional analysis of intraocular pressure at 6 months post surgery according to type of implant

\begin{tabular}{llll}
\hline $\begin{array}{l}\text { IOP } \\
\text { threshold } \\
\text { at } 6 \text { months }\end{array}$ & $\begin{array}{l}\text { iStent } \\
\text { device } \\
(\%)\end{array}$ & $\begin{array}{l}\text { iStent } \\
\text { inject } \\
\text { device } \\
(\%)\end{array}$ & $\begin{array}{l}\boldsymbol{p} \text { value } \\
\text { group comparison } \\
\text { of percentage of } \\
\text { eyes at each IOP } \\
\text { threshold) }\end{array}$ \\
\hline IOP $<18 \mathrm{mmHg}$ & 86.8 & 100.0 & 0.033 \\
IOP $<16 \mathrm{mmHg}$ & 84.2 & 88.6 & 0.422 \\
IOP $<14 \mathrm{mmHg}$ & 55.3 & 71.4 & 0.118 \\
IOP $<12 \mathrm{mmHg}$ & 7.9 & 25.7 & 0.040 \\
\hline
\end{tabular}

a Chi-square test (comparison between groups)

\section{Safety}

Both implant groups demonstrated favorable safety. Intraoperatively, there were two cases of technical difficulty with stent implantation (iStent eyes) that resulted in no sequelae and two cases of mild hemorrhagic reflux at the implantation site that resolved without intervention by postoperative Week 1 . No eyes with the iStent inject device experienced an intraoperative complication (difference in intraoperative complication rate between groups: $p=0.142$, not significant). Gonioscopy was performed intraoperatively and at all postoperative examinations; no significant problems in stent position were identified in either group at any point during the study. During the entire 6-month follow-up, all eyes in both groups maintained or improved their visual acuity versus baseline (preoperative), which is consistent with expectations for phacoemulsification cataract surgery. A minority of eyes (42.9\%) presented a visual acuity of $20 / 30$ or better at baseline, whereas $85.7 \%$ had that visual acuity level at the 6-month follow-up time point.

Only one eye $(2.6 \%)$ with the iStent implant had an adverse event during the entire 6 months of follow-up. This one eye had

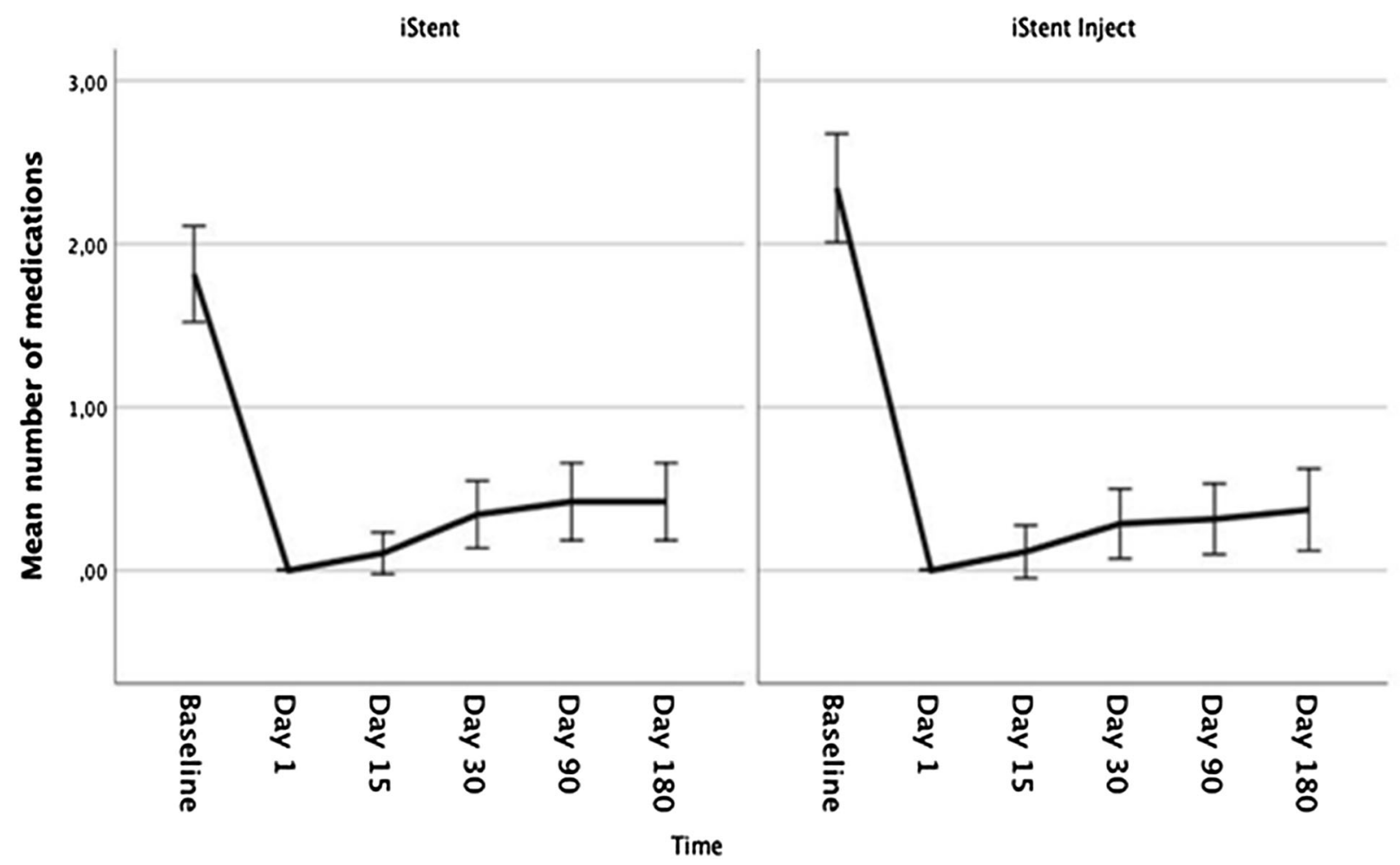

Fig. 4 Mean number of medications from baseline to 6 months (Day 180) post surgery according to type of implant (iStent, $n=38$ eyes; iStent inject, $n=35$ ). $p<0.001$ at all postoperative visits in both groups. Vertical bars represent SE 


\section{a}

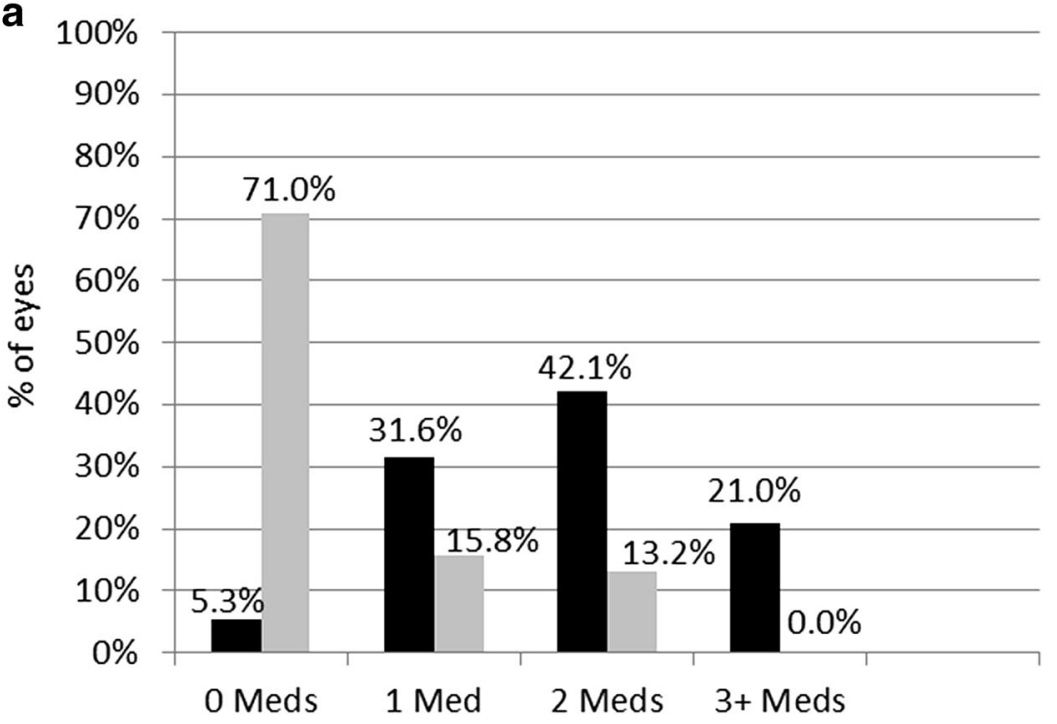

- Preoperative

Month 6

b



Fig. 5 Proportional analysis of medication use at baseline (Preoperative) and at 6 months (Day 180) post surgery. a iStent group ( $n=38$ eyes), $\mathbf{b}$ iStent inject group $(n=35$ eyes)

peripheral anterior synechiae resulting in stent occlusion at 3 months post surgery, which was corrected in-office by Nd:YAG laser iridotomy and resulted in no further sequelae. No adverse events were reported for eyes with the iStent inject implant.

During the follow-up, two eyes with the iStent implant underwent secondary surgery (non-penetrating deep sclerectomy). In one of these eyes, the IOP became elevated after systemic steroid medication was given for asthma; no associated stent malfunction was noted. The IOP remained elevated in this eye despite topical medications, and systemic steroid withdrawal was not medically safe, so the decision was made to undertake surgery. In the second eye, IOP elevation occurred at approximately 2 months post surgery and did not have any 
known cause or stent malfunction. The patient had dry eye and medication hypersensitivities, so surgery was considered instead of additional medication to manage the IOP. There were no secondary surgeries in the iStent inject group.

\section{DISCUSSION}

This real-world, single-surgeon study provides the first side-by-side comparative data on iStent and iStent inject devices in eyes with glaucoma. Outcomes for 6 months following surgery showed favorable safety and meaningful reductions in IOP and medication, with a directional trend toward better outcomes with the iStent inject device versus the iStent device. These findings were achieved in a real-world clinical setting and included all-comers, rather than a selection of only certain patients to yield positive outcomes. This approach allows for greater generalizability to physicians and patients seeking a suitable glaucoma treatment.

Clinically and statistically significant IOP reductions from baseline were observed in both implant groups at Month $6(p<0.001)$. These reductions are particularly notable given the relatively well-controlled IOP in both groups preoperatively (16.5 and $17.3 \mathrm{mmHg}$, respectively), which predisposes to modest postoperative IOP reductions [30].

The postoperative 6-month IOP range of approximately $12.5-14.0 \mathrm{mmHg}$ is also significant given that these ab-interno (inside the eye) stent procedures act on the trabecular outflow pathway, where IOP reaches the physiologic lower limit of episcleral venous pressure. The potential for these trabecular stents to produce IOP reduction is not expected to be as dramatic as suprachoroidal or subconjunctival procedures; however, their trabecular location also underlies their lower safety risks compared to suprachoroidal or subconjunctival surgeries.

Although our limited sample size prevents us from drawing definitive conclusions, directional trends can be discerned in our comparison of the postoperative IOP and medications for the two groups. The IOP reduction in eyes with the iStent inject implant was approximately double that of those eyes receiving the
iStent implant (26.6 vs. $15.8 \%$, respectively; $p=0.005)$. Several factors may contribute to this significant difference. As shown in preclinical studies, and subsequently borne out in the clinical arena [33, 39, 40, 44-47], the greatest reduction in IOP and outflow resistance comes from the first stent, with additional stents resulting in incrementally greater reductions in outflow resistance and IOP beyond those achieved by the first.

Both groups in our study were able to reduce their medication burden by over $80 \%$, with a decrease of 1.4 medications in the iStent group and a decrease of 1.9 medications in the iStent inject group. The medication regimen from preoperative (baseline) to 6 months post surgery was either decreased or maintained in all eyes in both groups, and over $70 \%$ of eyes in both groups became medication-free by 6 months. The most salient benefits of reducing medication reliance are threefold: (1) medication effectiveness is dependent upon treatment adherence, which is known to be low in the majority of glaucoma patients; (2) topical medications result in local and systemic side effects and ocular surface compromise; (3) and medications carry costs associated with the drops themselves and with the additional caregiving and office visits needed to monitor medication regimens [6-10]. This latter drawback has been included in several recent population-based cost-effectiveness analyses of iStent surgery, which have shown the durable cost-benefit of stent surgery versus alternative treatment options [48-51].

The safety profile of both groups was excellent, corroborating the existing body of evidence showing favorable safety of these devices [15-43]. All eyes maintained or improved visual acuity from their preoperative (baseline) levels, suggesting no negative impact of stent implantation upon the visual improvement expected with cataract surgery. There were no reports of the complications seen with filtering surgeries, such as hypotony, choroidal detachment, endophthalmitis, bleb-related complications, or infection [12-14]. During the 6 months of postoperative follow-up, adverse events occurred in the iStent group only, and these were limited and resulted in no sequelae. Two eyes 
with iStent implants had secondary surgery (non-penetrating deep sclerectomy) due to IOP elevation with no associated stent malfunction. There were no adverse events or secondary surgeries recorded in the iStent inject group.

Although both the iStent and iStent inject devices performed well in this cohort of eyes, the safety and effectiveness outcomes in the two groups suggest a treatment advantage favoring iStent inject over iStent. This advantage may be attributed to several characteristics of the device design. First, the presence of two stents, rather than one, in the trabecular meshwork allows for greater IOP-reducing potential, as shown in prior clinical and preclinical studies. Second, each individual iStent inject stent has four lateral outflow lumens, with the aim to maximize the number of clock-hours accessed for outflow. Finally, the iStent inject implantation procedure is considered to be more straightforward and efficient than that of the iStent, leading to a flatter and shorter learning curve for surgeons. This procedural elegance also conceivably allows for fewer complications with the iStent inject device than with the iStent device, as was observed in this study.

Because all patients underwent combined trabecular microbypass stent-cataract surgery, it was not possible to separate the IOP effect of cataract extraction versus stent implantation. Thus, patients' postoperative outcomes should be understood in the context of expected IOP reductions after phacoemulsification alone. As established in the literature, post-phacoemulsification IOP reduction is estimated to be $<2 \mathrm{mmHg}[52,53]$ and/or to be $16.5 \%$ reduced [54] versus baseline IOP. Eyes with higher preoperative IOP are known to have greater postoperative IOP reduction [54]. In the present study, the majority of eyes had early glaucoma and relatively well-controlled preoperative IOP (16.5 and $17.3 \mathrm{mmHg}$ in the iStent and iStent inject eyes, respectively), thereby predisposing to smaller reductions during the postoperative period. Despite this modest expectation, the study observed IOP reductions of $2.6-4.6 \mathrm{mmHg}$ in the two groups $(p<0.001$ for both); and equally importantly, these IOP reductions were achieved while also reducing the medication burden by $1.4-1.9$ medications in the two groups ( $p<0.001$ for both).

Several limitations to our study should be mentioned. There was a modest number of eyes in each group. The study was intended to be a retrospective, non-comparative, preliminary outcomes analysis for iStent and iStent inject technologies in Brazil; however, future research could include prospective comparative trials, trials with larger sample sizes, and/or multicenter trials. All surgeries were completed by a single surgeon, and the groups were drawn from the surgeon's real-life clinical population and thus were not identical; however, this realworld format may enhance applicability to current clinical settings by including a heterogeneous patient base unconstrained by the inclusion/exclusion criteria of traditional product-registration trials. There was no control group of cataract surgery only for comparison to the stent-cataract surgery groups. However, given that IOP and medications are numeric measurements, and medication decisions were made by the same doctor before and after surgery, patients' preoperative IOP and medication number may be considered to be valid comparators for postoperative values. No medication washouts were undertaken, as this could compromise the ocular health of these realworld glaucoma patients. However, given that pre- and post-operative IOP and medications were reported in the same eyes, patients' preoperative values were considered to be reasonable comparators for postoperative efficacy. This study covers outcomes during a 6-month postoperative period, as the iStent and iStent inject devices are relatively new technologies in Brazil. Thus, the results represent some of the first available data available in Brazil and for this surgeon. Future reports may include data with longer periods of follow-up.

\section{CONCLUSIONS}

These preliminary real-world data demonstrate significant reductions in IOP and number of anti-glaucomatous eye drops after implantation of either the iStent or iStent inject trabecular micro-bypass stent model. Implantation of the 
iStent inject device led to higher relative reductions in IOP and medication number and fewer adverse events versus implantation of the iStent device. A prospective study with a larger population and longer follow-up is necessary to validate these findings.

\section{ACKNOWLEDGEMENTS}

We thank the participants of the study.

Funding. No financial sponsorship was received for the work in this study. Article processing charges were funded by Glaukos Corporation. All authors had full access to all of the data in this study and take complete responsibility for the integrity of the data and accuracy of the data analysis.

Editorial Assistance. Editorial Assistance in the form of Medical Writing was conducted by Dr Dana Hornbeak from Glaukos Inc. Since all authors of the manuscript are native in the Portuguese language, Dr Dana Hornbeak had the courtesy to revise the English language in the text. Neither Dr. Hornbeak or Glaukos Inc. charged us for doing this service.

Authorship. All named authors meet the International Committee of Medical Journal Editors (ICMJE) criteria for authorship for this article, take responsibility for the integrity of the work as a whole, and have given their approval for this version to be published.

Disclosures. Ricardo Augusto Paletta Guedes has the following disclosures: consultant to Glaukos; Alcon; Allergan; OphtaVision Brasil. Daniela Marcelo Gravina, Jonathan Clive Lake, Vanessa Maria Paletta Guedes and Alfredo Chaoubah have nothing to disclose.

Compliance with Ethics Guidelines. All procedures were in accordance with the Institutional Review Board of the Paletta Guedes Eye Institute and with the 1964 Helsinki declaration and its later amendments or comparable ethical standards. Given that this was a retrospective outcomes analysis, including only patients from the surgeon's real-world clinical population who already had received treatment, formal clinical trial registration was not required. As it was a retrospective study, based on information on the patients' medical records, informed consent from study participants was not necessary.

Data Availability. The datasets generated during and/or analyzed during the current study are available from the corresponding author on reasonable request.

Open Access. This article is distributed under the terms of the Creative Commons Attribution-NonCommercial 4.0 International License (http://creativecommons.org/licenses/ by-nc/4.0/), which permits any noncommercial use, distribution, and reproduction in any medium, provided you give appropriate credit to the original author(s) and the source, provide a link to the Creative Commons license, and indicate if changes were made.

\section{REFERENCES}

1. Leske MC, Heijl A, Hyman L, Bengtsson B, Komaroff E. Factors for progression and glaucoma treatment: the Early Manifest Glaucoma Trial. Curr Opin Ophthalmol. 2004;15:102-6.

2. Heijl A, Leske MC, Bengtsson B, et al. Reduction of intraocular pressure and glaucoma progression: results from the Early Manifest Glaucoma Trial. Arch Ophthalmol. 2002;120:1268-1279.

3. Kass MA, Heuer DK, Higginbotham EJ, et al. The Ocular Hypertension Treatment Study: a randomized trial determines that topical ocular hypotensive medication delays or prevents the onset of primary open-angle glaucoma. Arch Ophthalmol 2002;120:701-713 (discussion 829-830).

4. The Advanced Glaucoma Intervention Study (AGIS): 7. The relationship between control of intraocular pressure and visual field deterioration. Am J Ophthalmol 2000;130:429-440.

5. American Academy of Ophthalmology. Preferred practice pattern: primary open-angle glaucoma (PDF file). 2015; pp 64-67, 73-74. https://www.aao. org/preferred-practice-pattern/primary-open-angleglaucoma-ppp-2015. Accessed 7 Dec 2018. 
6. Nordstrom BL, Friedman DS, Mozaffari E, Quigley HA, Walker AM. Persistence and adherence with topical glaucoma therapy. Am J Ophthalmol. 2005;140(4):598-606.

7. Tsai JC. A comprehensive perspective on patient adherence to topical glaucoma therapy. Ophthalmology. 2009;116:S30-6.

8. Newman-Casey PA, Robin AL, Blachley T, et al. The most common barriers to glaucoma medication adherence: a cross-sectional survey. Ophthalmology. 2015;122(7):1308-16.

9. Baudouin C, Liang H, Hamard P, et al. The ocular surface of glaucoma patients treated over the long term expresses inflammatory markers related to both T-helper 1 and T-helper 2 pathways. Ophthalmology. 2008;115(1):109-15.

10. Leung EW, Medeiros FA, Weinreb RN. Prevalence of ocular surface disease in glaucoma patients. J Glaucoma. 2008;17(5):350-5.

11. Glaucoma Laser Trial Research Group. The Glaucoma Laser Trial (GLT) and glaucoma laser trial follow-up study: 7 Results. Am J Ophthalmol. $1995 ; 120: 718-31$.

12. Gedde SJ, Herndon LW, Brandt JD, Budenz DL, Feuer WJ, Schiffman JC; Tube Versus Trabeculectomy Study Group. Postoperative complications in the Tube Versus Trabeculectomy (TVT) study during five years of follow-up. Am J Ophthalmol. 2012;153:804-814.

13. Rulli E, Biagioli E, Riva I, et al. Efficacy and safety of trabeculectomy vs nonpenetrating surgical procedures: a systematic review and meta-analysis. JAMA Ophthalmol. 2013;131(12):1573-82.

14. Jampel HD, Musch DC, Gillespie BW, Lichter PR, Wright MM, Guire KE; Collaborative Initial Glaucoma Treatment Study Group. Collaborative Initial Glaucoma Treatment Study Group. Perioperative complications of trabeculectomy in the collaborative initial glaucoma treatment study (CIGTS). Am J Ophthalmol. 2005;140(1):16-22.

15. Samuelson TW, Katz LJ, Wells JM, et al. Randomized evaluation of the trabecular micro-bypass stent with phacoemulsification in patients with glaucoma and cataract. Ophthalmol. 2011;118:459-67.

16. Craven ER, Katz LJ, Wells JM, Giamporcaro JE, iStent Study G. Cataract surgery with trabecular micro-bypass stent implantation in patients with mild-to-moderate open-angle glaucoma and cataract: two-year follow-up. J Cataract Refract Surg. 2012;38(8):1339-1345.
17. Arriola-Villalobos P, Martinez-de-la-Casa JM, DiazValle D, et al. Combined iStent trabecular microbypass stent implantation and phacoemulsification for coexisting open-angle glaucoma and cataract: a long-term study. Br J Ophthalmol. 2012;96:645-9.

18. Arriola-Villalobos P, Martinez-de-la-Casa JM, DiazValle D, Morales-Fernandez L, Fernandez-Perez C, Garcia-Feijoo J. Glaukos iStent inject ${ }^{\circledR}$ trabecular micro-bypass implantation associated with cataract surgery in patients with coexisting cataract and open-angle glaucoma or ocular hypertension: a long-term study. J Ophthalmol. 2016;2016:1056573.

19. Fea AM. Phacoemulsification versus phacoemulsification with micro-bypass stent implantation in primary open-angle glaucoma. J Cataract Refract Surg. 2010;36:407-12.

20. Fea AM, Belda JI, Rękas M, et al. Prospective unmasked randomized evaluation of the iStent inject ${ }^{\circledR}$ versus two ocular hypotensive agents in patients with primary open-angle glaucoma. Clin Ophthalmol. 2014;8:875-82.

21. Fea AM, Consolandi G, Zola M, et al. Micro-Bypass implantation for primary open-angle glaucoma combined with phacoemulsification: 4-year followup. J Ophthalmol. 2015;26(2015):795357.

22. Donnenfeld ED, Solomon KD, Voskanyan L, et al.. A prospective 3-year follow-up trial of implantation of two trabecular microbypass stents in open-angle glaucoma. Clin Ophthalmol. 2015;9:2057-65.

23. Chang DF, Donnenfeld ED, Katz LJ, et al. Efficacy of two trabecular micro-bypass stents combined with topical travoprost in open-angle glaucoma not controlled on two preoperative medications: 3-year follow-up. Clin Ophthalmol. 2017;11:523-8.

24. Voskanyan L, Garćia-Feijoo J, Belda JI, Fea A, Junemann A, Baudouin C. Synergy Study Group. Prospective, unmasked evaluation of the iStent ${ }^{\circledR}$ inject system for open-angle glaucoma: synergy trial. Adv Ther. 2014; 31(2):189-201.

25. Ferguson T, Swan R, Ibach M, Schweitzer J, Sudhagoni R, Berdahl JP. Evaluation of a trabecular microbypass stent with cataract extraction in severe primary open-angle glaucoma. J Glaucoma. 2018;27(1):71-6.

26. Myers JS, Masood I, Hornbeak DM, et al. Prospective evaluation of two iStent trabecular stents, one iStent Supra suprachoroidal stent and postoperative prostaglandina in refractory glaucoma: 4-year outcomes. Adv Ther. 2018;35:395-407.

27. Gallardo MJ, Supnet RA, Giamporcaro JE, Hornbeak DM. Outcomes of combined trabecular micro- 
bypass and phacoemulsification in a predominantly Hispanic patient population. Clin Ophthalmol. 2016;10:1931-7.

28. Neuhann TH. Trabecular micro-bypass stent implantation during small incision cataract surgery for open angle glaucoma or ocular hypertension: long term results. J Cataract Refract Surg. $2015 ; 41: 2664-71$.

29. Ferguson TJ, Swan R, Ibach M, Schweitzer J, Sudhagoni $\mathrm{R}$, Berdahl JP. Trabecular microbypass stent implantation with cataract extraction in pseudoexfoliation glaucoma. J Cataract Refract Surg. 2017;43(5):622-6.

30. Ferguson TJ, Berdakl JP, Schweitzer JA, Sudhagoni RG. Clinical evaluation of a trabecular micro-bypass stent with phacoemulsification in patients with open-angle glaucoma and cataract. Clin Ophthamol. 2016;10:1767-73.

31. Ferguson T, Berdahl J, Schweitzer J, Sudhagoni R. Evaluation of a trabecular micro- bypass stent in pseudophakic patients with open-angle glaucoma. J Glaucoma. 2016;25:896-900.

32. Vold SD, Voskanyan L, Tetz M, et al. Newly diagnosed primary open-angle glaucoma randomized to 2 trabecular bypass stents or prostaglandin: outcomes through 36 months. Ophthalmol Ther. 2016;5(2):161-72.

33. Katz LJ, Erb C, Carceller Guillamet A, . Long-term tirated IOP control with one, two or three trabecular micro-bypass stents in open-angle glaucoma subjetcs on topical hypotensive medication: 42-month outcomes. Clin Ophthalmol. 2018;12:255-62.

34. Klamann MK, Gonnermann J, Pahlitzsch M, et al. iStent inject in phakic open angle glaucoma. Graefes Arch Clin Exp Ophthalmol. 2015;253:941-7.

35. Seibold LK, Gamett KM, Kennedy JB, et al.. Outcomes after combined phacoemulsification and trabecular microbypass stent implantation in controlled open-angle glaucoma. J Cataract Refract Surg. 2016;42:1332-8.

36. Berdahl J, Voskanyan L, Myers JS, et al. Implantation of two second-generation trabecular microbypass stents and topical travoprost in open-angle glaucoma not controlled on two preoperative medications: 18-month follow-up. Clin Exp Ophthalmol. 2017;45(8):797-802.

37. Lindstrom R, Lewis R, Hornbeak DM, et al. Outcomes following implantation of two second-generation trabecular micro-bypass stents in patients with open-angle glaucoma on one medication: 18-month follow-up. Adv Ther. 2016;33(11):2082-90.

38. Hengerer FH, Auffarth GU, Riffel C, Conrad-Hengerer I. Prospective, non-randomized, 36-month study of second-generation trabecular micro-bypass stents with phacoemulsification in various types of glaucoma. Ophthalmol Ther. 2018;7(2):405-15. .

39. Belovay GW, Naqi A, Chan BJ, Rateb M, Ahmed II. Using multiple trabecular micro-bypass stents in cataract patients to treat open-angle glaucoma. J Cataract Refract Surg. 2012;38(11):1911-7.

40. El Wardani M, Bergin C, Achache F, Sharkawi E. Evaluating the trabecular micro-bypass stent combined with phacoemulsification compared to phacoemulsification alone. Klin Monatsbl Augenheilkd. 2015;232:442-5.

41. Malvankar-Mehta MS, Chen YN, Iordanous Y, Wang WW, Costella J, Hutnik CM. iStent as a solo procedure for glaucoma patients: a systematic review and meta-analysis. PLoS One. 2015;10(5):e0128146.

42. Malvankar-Mehta MS, Iordanous Y, Chen YN, et al. iStent with phacoemulsification versus phacoemulsification alone for patients with glaucoma and cataract: a meta-analysis. PLoS One. 2015;10(7):e0131770.

43. Popovic M, Campos-Moller X, Saheb H, K Ahmed II. Efficacy and adverse event profile of the iStent and iStent inject trabecular micro-bypass for open-angle glaucoma: a meta-analysis. J Curr Glaucoma Pract. 2018;12(2):67-84.

44. Hunter $\mathrm{K}$, Fjield $\mathrm{T}$, Heitzmann $\mathrm{H}$, Shandas $\mathrm{R}$, Kahook M. Characterization of micro-invasive trabecular bypass stents by ex vivo perfusion and computational flow modeling. Clin Ophthalmol. 2014;8:499-506.

45. Bahler CK, Smedley GT, Zhou J, Johnson DH. Trabecular bypass stents decrease intraocular pressure in cultured human anterior segments. Am J Ophthal. 2004;138(6):988-94.

46. Bahler C, Hann C, Fjield T, Haffner D, Heitzmann $\mathrm{H}$, Fautsch MP. Second-generation trabecular meshwork bypass stent (iStent inject) increases outflow facility in cultured human anterior segments. Am J Ophthal. 2012;153(6):1206-13.

47. Johnstone MA. The aqueous outflow system as a mechanical pump: evidence from examination of tissue and aqueous movement in human and nonhuman primates. J Glaucoma. 2004;13(5):421-38.

48. Ordóñez JE, Ordóñez A, Osorio UM. Cost-effectiveness analysis of iStent trabecular micro-bypass 
stent for patients with open-angle glaucoma in Colombia. Curr Med Res Opin. 2018;10:1-12.

49. Tan SZ, Au L. Manchester iStent study: 3-year results and cost analysis. Eye (Lond). 2016;30(10):1365-70.

50. Berdahl JP, Khatana AK, Katz LJ, Herndon L, Layton AJ, Yu TM, Bauer MJ, Cantor LB. Cost-comparison of two trabecular micro-bypass stents versus selective laser trabeculoplasty or medications only for intraocular pressure control for patients with openangle glaucoma. J Med Econ. 2017;20(7):760-6.

51. Ngan K, Fraser E, Buller S, Buller A. A cost minimization analysis comparing iStent accompanying cataract surgery and selective laser trabeculoplasty versus topical glaucoma medications in a public healthcare setting in New Zealand. Graefes Arch Clin Exp Ophthalmol. 2018;256(11):2181-9.

52. Vizzeri G, Weinreb RN. Cataract surgery and glaucoma. Curr Opin Ophthalmol. 2010;21(1):20-4.

53. Friedman DS, Jampel HD, Lubomski LH, et al. Surgical strategies for coexisting glaucoma and cataract: an evidence-based update. Ophthalmology. 2002;109:1902-13.

54. Mansberger SL, Gordon MO, Jampel $\mathrm{H}$, et al. Reduction in intraocular pressure after cataract extraction: the Ocular Hypertension Treatment Study. Ophthalmology. 2012;119:1826-31. 